\title{
Past 24 Hours
}

National Cancer Institute

\section{Source}

National Cancer Institute. Past 24 Hours. NCI Thesaurus. Code C118250.

The 24 hour period occurring just before the present time. 